\title{
O Problema de Coordenação em um Algoritmo de Controle em Tempo Real de Tráfego Urbano
}

\section{The Coordination Problem in an Real Time Control Algorithm of Urban Traffic}

\author{
Silvia G. S. Cervantes ${ }^{1}$; Werner Kraus Junior ${ }^{2}$
}

\section{Resumo}

Apresenta-se a otimização do controle semafórico do tráfego urbano, em tempo real, usando busca em profundidade em grafos. O modelo de tráfego veicular usado na predição do comportamento futuro do tráfego é descrito por equações de estados. O método de controle fornece, a partir de contagens de fluxo veicular, os tempos de abertura dos semáforos que resultem no melhor desempenho possível para a malha viária e que proporcione ainda uma coordenação de fluxos entre interseções. A medida deste desempenho é o atraso médio dos veículos. O algoritmo é comparado com uma estratégia de tempo fixo ajustado pelo programa TRANSYT mostrando desempenho superior.

Palavras-chave: Controle de Tráfego urbano. Otimização. Controle Adaptativo.

\begin{abstract}
The optimization of the signal control of the urban traffic in real time is presented, using depth search procedure in graphs. The traffic of vehicles model used for the prediction of future traffic behavior is described by means of state equations. The control method provides signal opening timings which result in the best possible performance for the transport system as well as a coordination of traffic flows between intersections. Performance is measured by the average delay of vehicles. The algorithm shows better performance when compared with a fixed time strategy with timings calculated by the TRANSYT program.
\end{abstract}

Key words: Urban Traffic Control. Optimization. Adaptive Control.

\footnotetext{
${ }^{1}$ Professora Adjunto do DEEL-UEL; silvia@uel.br

${ }^{2}$ Professor Adjunto I da Universidade Federal de Santa Catarina UFSC; werner@das.ufsc.br
} 


\section{Introdução}

Do ponto de vista do cálculo dos tempos, o controle semafórico pode ser dividido em duas categorias: as que utilizam planos de tempos fixos, selecionados por horário ou por volume de tráfego, e as que calculam a temporização em tempo real. Estes últimos, apesar de terem custo maior de implantação, são preferíveis, pois conseguem ajustar-se automaticamente às variações de fluxo e eliminam o custo de atualização dos planos de tempos fixos (WILSHIRE et al., 1985). Além disso, apresentam melhor desempenho, pois podem aproveitar-se de variações de demanda ciclo-a-ciclo, algo que não pode ser feito pelas estratégias de tempos fixos.

Os sistemas de controle capazes de reagir às variações de demanda de tráfego são objeto de grande interesse comercial e acadêmico. O produto mais bem sucedido é o SCOOT (ROBERTSON; BRETHERTON, 1991), que implementa um algoritmo similar ao do programa de tempos fixos TRANSYT (ROBERTSON, 1968), adaptado para funcionamento em tempo real. Muitos são os trabalhos dedicados ao estudo desta técnica; entre eles pode-se citar SCATS (LOWRIE, 1990), PRODYN (FARGES; KAMDEM; LESORT, 1991), OPAC (GARTNER, 1983), RHODES (MIRCHANDANI; HEAD, 2001), ALLONS-D (PORCHE et al., 1996).

Este trabalho apresenta um algoritmo de controle em tempo real, baseado em algoritmo de busca em profundidade modificado associado à estratégia de horizonte deslizante para otimização dos tempos semafóricos. Assim como em outros algoritmos da literatura, como PRODYN (FARGES; KAMDEM; LESORT, 1991) e OPAC (GARTNER, 1983), propõe-se a construção de uma árvore de decisão com base em modelo simplificado do tráfego (GERLOUGH; HUBER, 1975). A busca nesta árvore no PRODYN e no OPAC é realizada através de programação dinâmica. O objetivo é a minimização de um critério de desempenho. O algoritmo de controle também realiza uma troca de informações, que proporciona uma coordenação implícita de fluxo entre as interseções.

Serão apresentados os resultados do algoritmo proposto, bem como seu desempenho frente ao algoritmo de tempo fixo, baseado em ajuste de acordo com Transyt (ROBERTSON, 1968).

\section{Descrição do Problema}

O problema de otimização consiste em dado o modelo dinâmico que representa os estados do tráfego urbano, encontrar a seqüência de valores de controle $u=\left\{u_{t+1}, u_{t+2}, \ldots, u_{t+K}\right\}$ que minimiza o critério de desempenho escolhido. A seguir, são apresentados tanto o modelo dinâmico como o problema de otimização.

\section{Modelo de Tráfego}

Apresenta-se o modelo de tráfego utilizado neste trabalho, para predição das condições de tráfego necessárias para o desenvolvimento do algoritmo de controle. Este modelo foi desenvolvido com base no modelo de tráfego do PRODYN (FARGES; KAMDEM; LESORT, 1991), constitui uma simplificação deste, pois não considera conversões à esquerda. Estas conversões não serão consideradas porque, no contexto brasileiro, não são comuns planos semafóricos que incluem conversões à esquerda permitidas e não protegidas. São considerados instantes de tempo $t$, (tempo de amostra), espaçados de um período constante $t_{s}$, no caso 4 segundos, ou seja: $t_{s}=t_{j+1}-t_{j}$. O fluxo de veículos em uma rede é modelado 
através de equações de estado. Há um grupo de equações de estados para interseções que inicialmente descrevem a evolução da indicação semafórica:

$$
v_{i}(t+1)=u_{i}(t), \quad i=1,2, \ldots, I
$$

onde $v_{i}(t) \in\{0,1\}$ é o estágio vigente na interseção $i$, durante o intervalo de tempo $\left(t_{j}, t_{j+1}\right) ; u_{i}(t) \in\{0,1\}$ é o valor do controle a ser aplicado no período subseqüente e $I$ é o número de interseções do sistema.

O tempo de vigência de um mesmo estágio, medido em termos de número de períodos sem mudança, é dado por:

$$
w_{i}(t+1)=\left\{\begin{array}{ccc}
w_{i}(t)+1 & \text { se } & u_{i}(t)=v_{i}(t) \\
0 & \text { se } & u_{i}(t) \neq v_{i}(t)
\end{array}\right.
$$

onde $w_{i}(t)$ é o número de períodos decorridos no estágio vigente.

Outro grupo de equações de estado descreve as variáveis que caracterizam as vias do sistema. $\mathrm{O}$ modelo vertical da fila, em um link, tem evolução temporal dada por:

$$
x_{l}(t+1)=\max \left\{0, x_{l}(t)+a_{l, 1}(t)-s_{l} m_{l}(t)\right\}
$$

onde $x_{l}(t)$ é a fila formada na linha de parada de um link $l ; a_{l, 1}(t)$ é número de veículos em velocidade livre que trafegam na primeira seção de um link; $s_{l}$ é o fluxo de saturação, isto é, a taxa média de descarga da fila, considerado conhecido e constante para todos os links e $m_{l}(t) \in\{0,1\}$ é o controle do link em questão, sendo $m_{l}(t)=$ $u_{i}(t)$ para os links arteriais e $m_{l}(t)=\overline{u_{i}(t)}$ para os links secundários.
As seções de um link são definidas por:

$$
\mathcal{N}(l)=\frac{L_{l}}{v_{l} t_{s}}
$$

onde $\mathcal{N}(l)$ é o número de seções de um link; $L_{l}$ é o comprimento de cada link, $v_{l}$ é a velocidade livre de percurso; e $t_{s}$ é o tamanho da amostra de tempo. $\mathcal{N}(l)$ é definido de tal forma que a passagem de uma seção para a seguinte é feita em exatamente um período amostral, como pode ser visto na Figura 1. A evolução da ocupação de veículos que trafegam em velocidade livre ao longo das seções de 1 a $N$ de um link, é dada por:

$$
\begin{aligned}
& a_{l, j}(t+1)=a_{l, j+1}(t), j=1, \ldots, N_{l}-1 \\
& a_{l, N_{l}}(t+1)=a_{l, N_{l}+1}(t)+\left(1-r_{l}\right) z_{l}(t) \\
& a_{l, N_{l}+1}(t+1)=r_{l} z_{l}(t)
\end{aligned}
$$

onde $z_{l}(t)$ é o fluxo de veículos no início do link, a montante da interseção; e $N_{l}$ é o número de períodos que um veículo gasta para atravessar um link, sendo $N_{l}$ a parte inteira e $r_{l}$ a parte decimal deste período.

Os fluxos de veículos $z_{l}(t)$ são modelados de duas formas. Para links alimentados por filas a montante, tem-se:

$$
z_{l}(t)=\sum_{l^{\prime} \in U_{l}} p_{l^{\prime} l} \times \min \left(x_{l^{\prime}}(t)+a_{l^{\prime}, 1}(t), s_{l^{\prime}} m_{l^{\prime}}(t)\right)
$$

onde $l^{\prime} \in U_{l}$, é o link pertencente a $U_{l}$ que é o conjunto de links que alimentam um determinado link $l$; $p_{l^{\prime} l}$ é a proporção de veículos que convergem das filas para o link a jusante, considerada conhecida e constante. Para os links de entrada, $z_{l}(t)$ é simplesmente o resultado da 


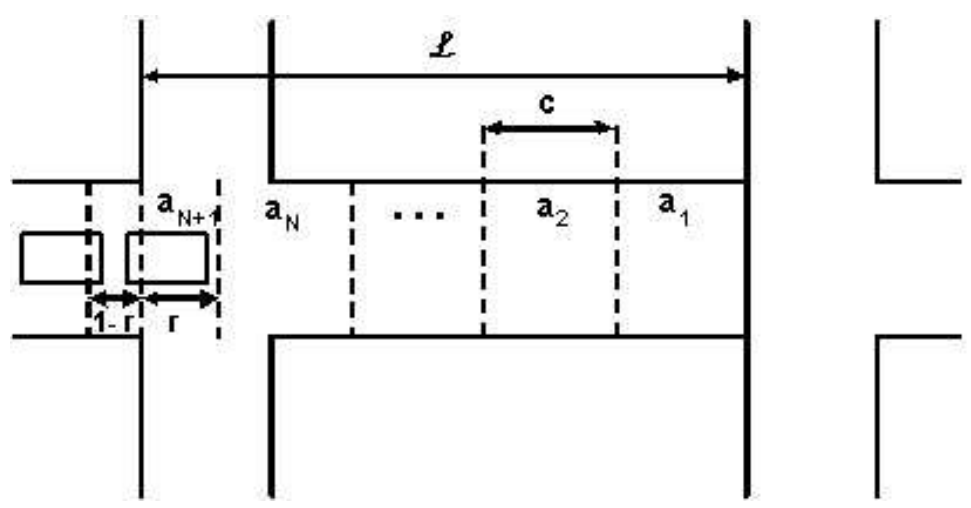

Figura 1: Seções de um link.

contagem veicular.

O desempenho da rede é avaliado por meio do atraso acumulado. O modelo de tráfego ao fornecer a dinâmica das filas, permite avaliar o atraso acumulado no conjunto de todos os links $l$ e em um horizonte $K+1$ :

$$
d_{l}(t)=\frac{t_{s}}{2} \sum_{j=t+1}^{t+K+1}\left(x_{l}(j)+x_{l}(j-1)\right)
$$

\section{Problema de Otimização}

O objetivo do algoritmo de controle proposto neste trabalho é a busca de uma solução ótima para os tempos de indicação semafórica. Um dos critérios de desempenho que pode ser utilizado para avaliação desta solução é o atraso global. Este atraso global pode ser representado, de forma simplificada, pela somatória das filas em um tempo infinito. Assim, o problema de otimização pode ser formulado como:

$P:$ Min $\quad f=\frac{t_{s}}{2} \sum_{i \in I} \sum_{l \in L_{i}} \sum_{t=1}^{T-1}\left\{x_{l}(t)+x_{l}(t+1)\right\}$

onde $L_{i} \subseteq E$ é o subconjunto dos links que chegam na interseção $i, \cup_{i \in I} L_{i}=E, L_{m} \cap L_{j}=$ $\emptyset, \forall m \neq j$ e $t_{s}$ é o tempo de amostragem sendo $t_{s}=t_{j+1}-t_{j}$, para $j=1, \ldots, T-1$. Além disso, $T$ é o número de períodos do horizonte de simulação considerado e $x_{l}(t)$ é o número de veículos estacionários na linha de parada do link l. Sujeito às restrições dadas pelas equações 3 , e de 5 a 8 e:

$$
u_{i}(t)=v_{i}(t) \quad \text { se } \quad w_{i}(t)<w_{\min }
$$

onde, $w_{\min }$ é o número mínimo exigido de permanência na mesma fase.

\section{Algoritmo de Controle}

A idéia central do algoritmo de controle consiste em testar todas as possibilidades de controle $u_{i}(t)$ em um horizonte de tempo e escolher dentre todas aquela que produz o melhor desempenho. Tal teste é efetuado com auxílio do modelo de tráfego apresentado na seção , o qual, ao fornecer a dinâmica das filas, permite avaliar os índices de desempenho, que neste trabalho é medido pelo atraso acumulado no conjunto de todas os links $l$ e em um horizonte $K+1$, conforme equação (9).

O algoritmo de controle utiliza a técnica de horizonte deslizante (NEWELL, 1998) para tentar prever o comportamento futuro do tráfego 
e descobrir o melhor controle para o futuro. A técnica consiste em simular, entre dois instantes de controle, a evolução do sistema durante vários períodos. Ao final, tem-se o valor ótimo do controle para ser aplicado, o qual irá vigorar apenas durante o período subseqüente. O processo é repetido para todos os períodos. As possibilidades criadas pelo horizonte deslizante podem ser representadas por uma árvore de decisão.

A árvore de decisão assim obtida é adequada para organizar a tarefa de testar todas as possibilidades. Ela é montada a partir das diferentes opções que uma decisão entre a indicação verde ou vermelha pode oferecer. Cada nova situação de tráfego criada tem um custo quantificado em atraso veicular. Um algoritmo de busca faz a tarefa de encontrar o caminho na árvore que resulte no menor custo. O resultado levará ao controle a ser aplicado no período subseqüente.

Uma simplificação assumida no modelo de tráfego e no algoritmo de controle é o modelo de fila vertical. Este modelo é utilizado em vários outros modelos já citados (FARGES; KAMDEM; LESORT, 1991), (GARTNER, 1982), (PORCHE; LAFORTUNE, 1997).

\section{Horizonte Deslizante}

O procedimento de horizonte deslizante pode ser descrito da seguinte forma: a técnica exige o conhecimento das chegadas de veículos no período inicial. Para os períodos futuros do horizonte são utilizadas predições das chegadas. Estas predições são obtidas para todas as possibilidades de controle durante um horizonte de tempo $K$, com $k=1, \ldots, K$. Este procedimento utiliza um modelo de predição, para este estudo, similar ao modelo de tráfego empregado. O tamanho do horizonte aplicado no algoritmo de controle em tempo real é $K=8$, ou seja, $K=8 t_{s}=32 \mathrm{~s}$.
O procedimento de otimização define o caminho de menor custo. Com este resultado definese o controle aplicado em $k=1$, neste caminho de menor custo. O controle em $k=1$ é implementado no período subseqüente e permanecerá vigente até que o procedimento seja repetido e novamente atualizado.

O resultado da aplicação do horizonte deslizante é representado por uma árvore de decisão. Esta árvore, para um semáforo de duas fases, é binária, pois, a decisão é tomada sobre uma variável discreta que pode assumir dois estados: a indicação verde ou vermelha. Os nós da árvore representam os estados do sistema. Suas arestas representam o custo da transição de um estado para outro. O custo da transição entre os estados é o atraso veicular, dado pela equação (9). Um exemplo de aplicação para um semáforo de dois estágios pode ser analisado na Figura 2.

A Figura 2 mostra uma árvore completa para $K=3$. A busca é realizada em profundidade, portanto é escolhido o ramo superior e percorrido o caminho até a última folha da árvore. $\mathrm{Na}$ Figura, o caminho totaliza um custo de 6 . A restrição de verde mínimo é cumprida, pois a indicação verde (g) é mantida durante todo o caminho. O segundo caminho a ser percorrido é o que testa a mudança para indicação vermelha (r), no segundo nível da árvore. Esta mudança é permitida, pois o verde mínimo já foi cumprido, no entanto, a partir deste ponto a restrição de verde mínimo é reiniciada então a folha imediatamente acima é inviável (folha marcada sem sombreamento). Já a folha imediatamente abaixo soma um custo de 6 . O procedimento é repetido para a metade inferior da árvore. A partir do primeiro nível, a mudança só é permitida para a folha acima, tornando todos os caminhos abaixo inviáveis. O custo resultante para a folha acima 


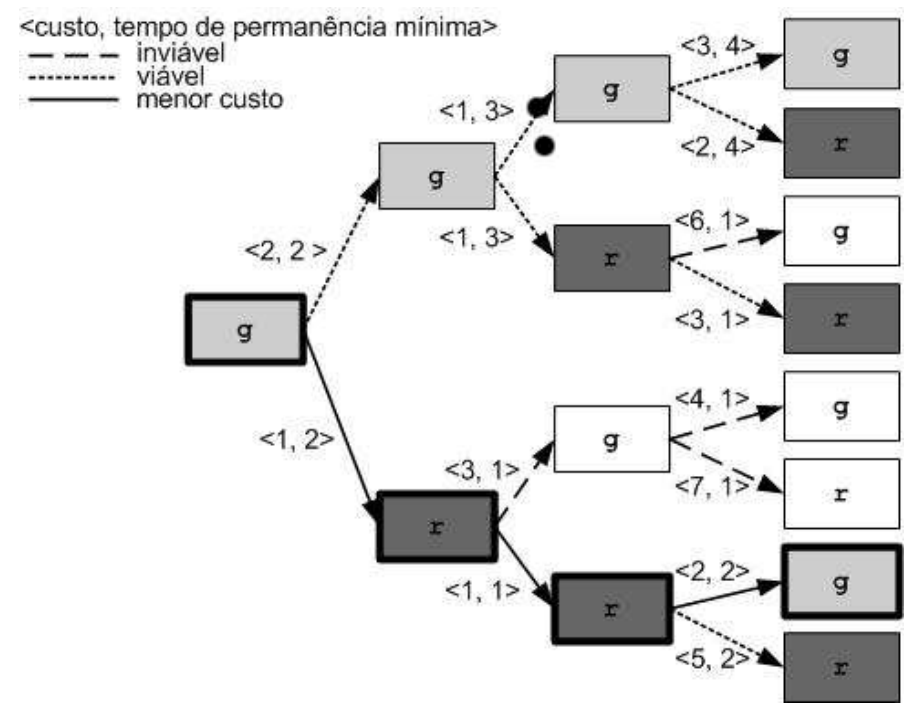

Figura 2: Árvore de busca - semáforo de dois estágios.

do segundo nível da árvore é 2 e o da folha abaixo 5. Assim, o caminho de menor custo (igual a 2) aparece realçado na figura 2 .

\section{Predição de Chegadas}

A predição de chegadas é realizada com o auxílio de um modelo de predição baseado no modelo de tráfego já apresentado. Algumas simplificações foram realizadas para tal aplicação, simulações de predição são realizadas o número de vezes que o horizonte é dividido em períodos; o controle que vigora no período inicial do horizonte, isto é, as indicações semafóricas de cada interseção, é considerado constante para todo o horizonte; o tipo de chegada pode ser definido pelo operador: a quantidade de veículos que chegam no primeiro período do horizonte é conhecida, resultante das contagens nos detectores. Para os períodos seguintes pode ser utilizada a média dos períodos anteriores (no tamanho igual ao número de períodos do horizonte) ou chegada nula de veículos.

\section{Heurística de Busca em Profundidade}

O algoritmo de busca utilizado é o de busca em profundidade (CORMEN et al., 2002). Esta escolha pode ser justificada pelos seguintes aspectos, simplicidade de implementação do método; a rapidez na obtenção de uma solução factível; pequeno consumo de memória durante a busca que é realizada em um caminho de cada vez, ao contrário, por exemplo da busca em largura, que precisa armazenar o valor do custo parcial de todas as folhas a cada nível que avança na árvore.

Por outro lado, existe a desvantagem da explosão combinatória de caminhos que crescem exponencialmente com o tamanho do horizonte. Esta desvantagem poderia inviabilizar a aplicação do algoritmo dependendo do tamanho do horizonte. Assim, algumas modificações foram implementadas ao algoritmo com o objetivo de restringir a busca em todos os caminhos de uma árvore.

A principal modificação é permitir que a decisão a ser tomada (permanecer no estágio atual ou mudar) possa ser explorada alternativamente na árvore. Isto é, a busca é realizada, inicial- 
mente, em uma direção da árvore, até atingir o último nível de profundidade, depois a busca retorna ao nó inicial e parte para a outra direção de busca. O valor do desempenho obtido no último nível da árvore, na direção inicial, é considerado o melhor até que uma busca em outro ramo qualquer (ao atingir o último nível) resulte em um valor de desempenho menor; neste ponto, invertem-se os papéis. Outra modificação é o descarte da busca em um determinado ramo ao ser obtido um valor de desempenho maior do que considerado ótimo até aquele momento da busca. Tal procedimento pode seguir até que seja realizada a busca em todos os ramos viáveis ou até que se atinja o limite de tempo disponível para o cálculo, quando então é adotada a decisão vencedora.

As modificações efetuadas também garantem a simulação das duas opções iniciais e distintas de controle, antes que um dead-line (FARINES; FRAGA; OLIVEIRA, 2000) possa ocorrer.

A busca na árvore tem outra restrição, além do dead-line, o tempo mínimo de duração da indicação semafórica dada pela equação (11). A necessidade desse limite mínimo é justificada pelo custo de mudança de fase, baseado no tempo mínimo para que os veículos que estejam parados em uma fila possam se deslocar através da interseção. Esta restrição inibe algumas mudanças de fase, poupando esforços computacionais na busca de um caminho de menor custo.

\section{Coordenação Implícita}

Quando interseções semaforizadas são localizadas nas proximidades de outras, o controle de cada interseção isoladamente interfere no comportamento de chegada de veículos nas outras interseções. Estas chegadas deixam de ser randômicas, pois passam a ocorrer na forma de pelotões nas interseções à jusante. Os pelotões são criados pela descarga da fila de veículos formada durante a indicação semafórica vermelha. Os pelotões tendem a sofrer dispersão, que depende do espaço entre as interseções semaforizadas, do comportamento do motorista e das condições de tráfego ao longo da via. Quando a distância entre interseções não suficientemente grande para que ocorra a dispersão, considera-se que o pelotão gerado na interseção à montante chega inalterado à interseção à jusante. Este comportamento motiva a tentativa de coordenar os semáforos de forma a privilegiar a passagem destes pelotões.

Verifica-se o estado ideal de coordenação entre interseções nas situações em que o primeiro veículo liberado durante o tempo de verde, atinge o final da fila na via à jusante no momento em que o último veículo desta fila está partindo da linha de retenção. Idealmente, este procedimento deve repetir-se entre as interseções adjacentes. Este fenômeno é ilustrado na Figura 3.

A coordenação semafórica, conhecida por onda verde, é uma coordenação que ocorre entre os tempos semafóricos com o objetivo de privilegiar a passagem de um dado pelotão. A coordenação que ocorre entre os semáforos não implica, necessariamente, a coordenação dos fluxos de veículos, ou seja, pode ocorrer uma dispersão do pelotão entre as interseções e, conseqüentemente, os veículos não chegarem a tempo de aproveitar o tempo de verde para cruzar a próxima interseção.

Em alguns algoritmos de Tempo Fixo e de Controle Atuado a variável offset pode ser uma variável de controle. Nesse caso, a coordenação semafórica pode ser obtida pelo ajuste desta 
variável entre interseções vizinhas. Em outros algoritmos de Controle Atuado o offset não aparece explicitamente, e portanto, não é controlado. Com isso, a coordenação é obtida de forma implícita, por meio da troca de informações a respeito das chegadas de veículos entre as interseções vizinhas durante o processo de otimização. Nos algoritmos de Controle Atuado, a coordenação também pode ser obtida de forma explícita, quando são criados níveis hierárquicos para o controle da coordenação. Um nível superior, que detém informações em nível de rede, define critérios para coordenação global.

A arquitetura descentralizada do modelo e do algoritmo de controle dificulta uma estratégia de coordenação entre as interseções. No entanto, como no PRODYN e ALLONS-D, a coordenação ocorre de forma implícita devido à incorporação de dados das interseções à montante da interseção analisada. Ou seja, quando os cálculos de otimização são realizados para uma interseção, são conhecidos os dados de saída da interseção acima dela considerando constante para todo o horizonte a indicação semafórica vigente. Dessa forma, a coordenação entre interseções é obtida implicitamente. Uma coordenação explícita também pode ser obtida através de restrições introduzidas ao algoritmo. Uma implementação desse tipo de coordenação, para o algoritmo de controle aqui proposto pode ser visto em (CARLSON, 2004).

\section{Resultados}

Para avaliar a consistência do algoritmo proposto inicialmente será apresentada uma comparação com a estratégia TRANSYT. O TRANSYT foi escolhido, pois é considerado uma estratégia padrão para cálculo de tempos fixos. Os resultados de desempenho do algoritmo de controle descentralizado serão apresentados de forma a avaliar sua sensibilidade frente a diferentes distribuições de demanda e chegadas.

\section{Características da Rede de testes}

Os testes foram realizados em uma rede de três interseções em um via arterial de sentido único de fluxo mostrada na Figura 4. O comprimento total da via arterial é de aproximadamente 760 m, divididos em 3 links de aproximadamente 253 metros. Os links secundários são de aproximadamente 350 metros. A velocidade dos veículos é considerada constante a um valor de 16, 7 metros/segundo $(60 \mathrm{~km} / \mathrm{h})$.

Serão apresentados resultados para diferentes instâncias de carregamento da rede apresentadas na Tabela 1. O carregamento considerado está abaixo do máximo grau de saturação, pois a concepção do algoritmo foi para controle de sistemas não-saturados. Diferentes distribuições de chegadas de veículos nas vias também foram consideradas. São quatro as distribuições: escada, exponencial, pulsada e constante. Foram realizadas três simulações distintas para cada distribuição na geração de dados de chegadas. Foi feita uma média aritmética dos valores obtidos, para cada distribuição.

As amostras de tempo do algoritmo são de 4 segundos. As proporções de propagação são consideradas conhecidas e iguais a $90 \%$ na arterial e $10 \%$ das secundárias para a arterial. O fluxo de saturação é igual a 2 veículos por amostra de tempo, também considerado conhecido e constante para todas as vias. A capacidade das vias é de 1800 veículos por hora (vph). E o horizonte $T$ de simulação $T=30 \mathrm{~min}$.

Serão testadas duas situações de carregamento, alto, $90 \%$ da capacidade da via, ou seja, 


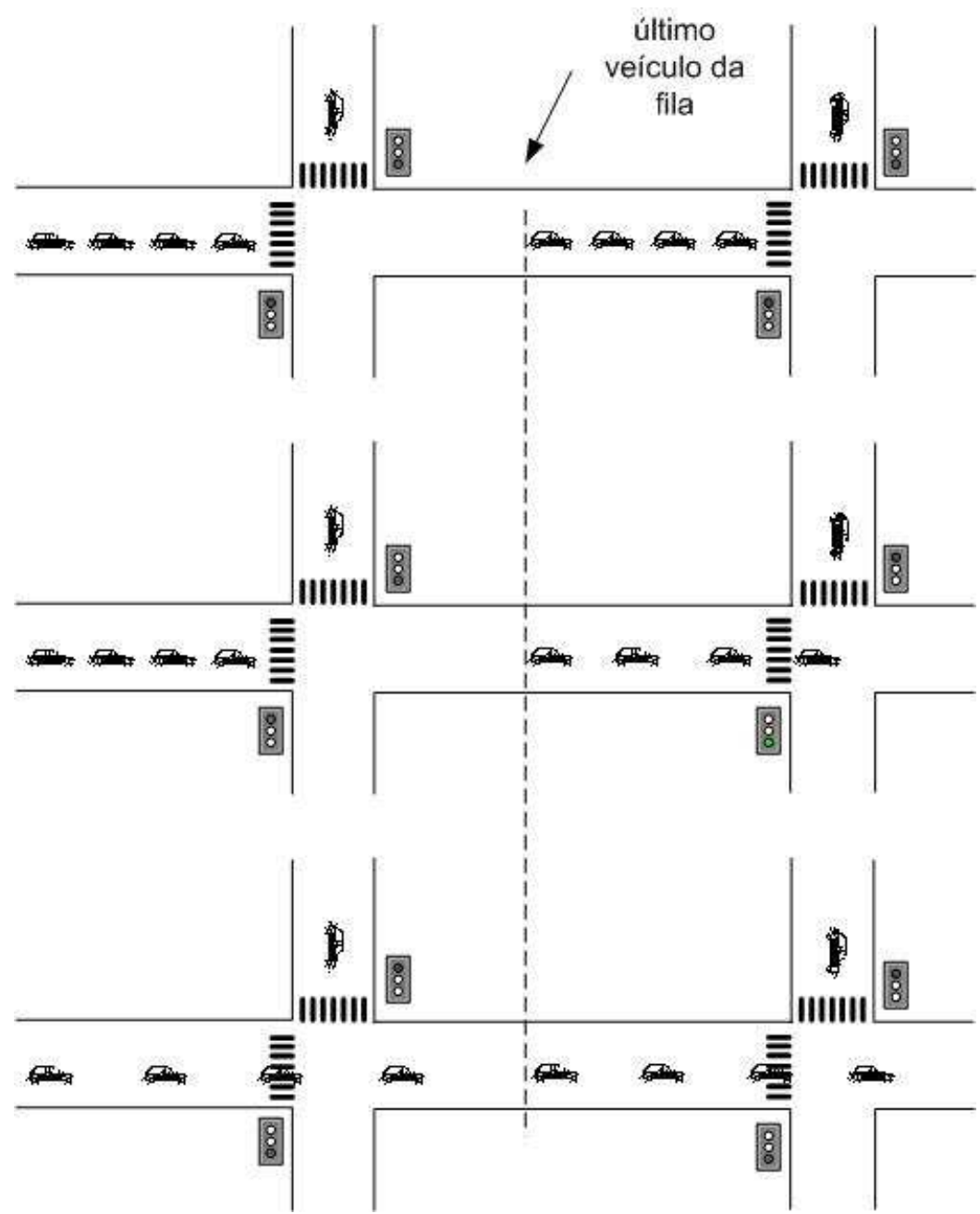

Figura 3: Coordenação entre interseções de uma via.

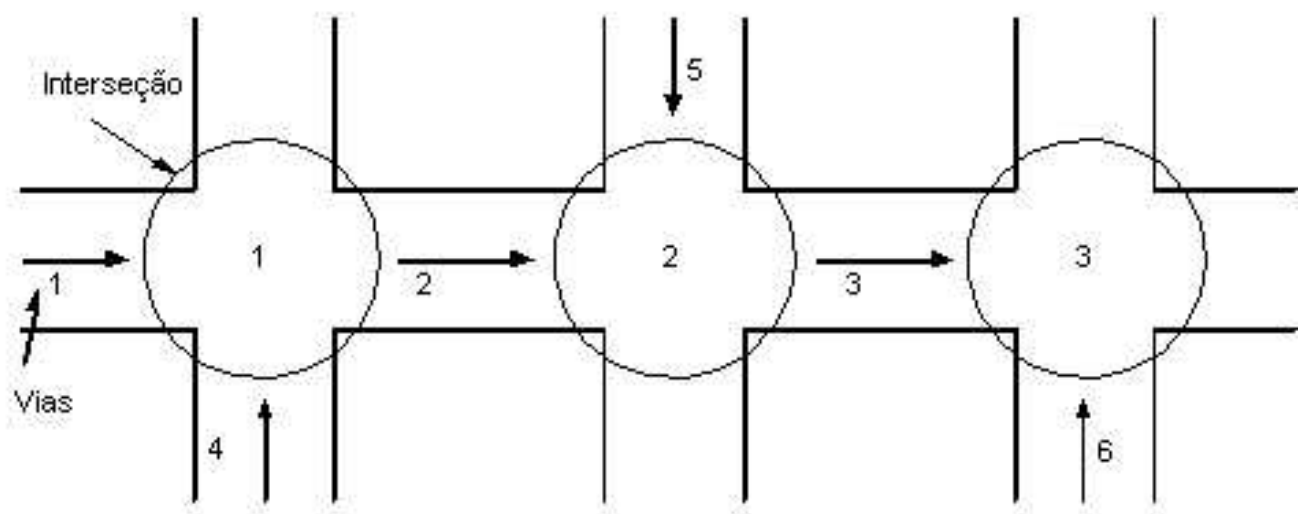

Figura 4: Rede de Tráfego para 3 interseções. 
Tabela 1: Proporção de fluxos nos links.

\begin{tabular}{ccc}
\hline \hline Caso & Arterial & Secundárias \\
\hline desbalanceado & $80 \%$ & $20 \%$ \\
balanceado & $55 \%$ & $45 \%$ \\
\hline
\end{tabular}

Tabela 2: Comparação com TRANSYT.

\begin{tabular}{lcc}
\hline \hline & TRANSYT & Desc. \\
\hline BA & 27,0 & 5,2 \\
DA & 20,0 & 6,6 \\
BM & 9,6 & 2,9 \\
DM & 6,9 & 3,1
\end{tabular}

onde: $\mathrm{BA}$ é balanceado alto, DA é desbalanceado alto, BM é balanceado médio e DM é desbalanceado médio.

um total de 1680 veículos por hora (vph); médio, $60 \%$ da capacidade da via, ou seja, um total de $1080 \mathrm{vph}$.

\section{Comparações com o TRANSYT}

Na Tabela 2, são apresentados na primeira coluna o valor do atraso obtido diretamente da simulação realizada com o TRANSYT em pcu-h/h e na segunda coluna são apresentados os valores obtidos diretamente do algoritmo de controle em veíc.-s/s Essas comparações foram realizadas com a distribuição de chegadas constante para satisfazer as especificações de entrada do TRANSYT.

A avaliação dos resultados apresentados na Tabela 2, permite verificar que o desempenho da rede obtido com o algoritmo em tempo real apresenta um ganho maior, o que era esperado pois, a otimização realizada procura responder à demanda verificada a cada amostra de tempo.

Outra comparação entre o desempenho obtido pelo TRANSYT e pelo algoritmo descentralizado pode ser verificado na Figura 5. É simulado um carregamento balanceado médio (BM) e desbalanceado médio (DM) e distribuição constante.
Pode ser verificada a evolução dos pelotões, ao longo do link arterial, e a formação das filas nas interseções.

Na Figura 5 (a), é apresentado o comportamento do tráfego frente ao controle realizado pelo algoritmo em tempo real e em (b) o comportamento frente ao controle fixo para um carregamento médio balanceado. Pode-se verificar que, em (b), os tempos de indicação verde e vermelha têm maior duração e que os ciclos são fixos. Foram testados valores de ciclo maiores e menores, no entanto o valor apresentado resulta em um melhor desempenho. Já em (a) os tempos de verde e vermelho são menores e adaptam-se à demanda verificada, e isso implica na menor formação de filas e, conseqüentemente em melhor desempenho. As filas são verificadas como picos que ocorrem nas interseções e os pelotões como uma progressão nas seções do link, verificada em (a) e (b). Em (c) e (d), apresentam-se os resultados para uma demanda média e desbalanceada. Verifica-se que, para um fluxo maior na arterial, o TRANSYT (d) proporciona uma melhoria de desempenho em relação a um fluxo balanceado entre os links (b). No entanto, o desempenho do TRANSYT ainda é pior que dos tempos calculados pelo algoritmo em tempo real (c). Outra particularidade que deve ser observada é o fato de a progressão de pelotões nas interseções serem verificadas pela formação de vales na Figura 5, identificando assim um bom resultado de coordenação entre interseções.

\section{Desempenho do Algoritmo de Controle em Tempo Real}

Nesta seção será apresentado o desempenho do algoritmo de controle em tempo real descentralizado para as quatro distribuições de chegadas (escada, exponencial, pulsada e constante) 


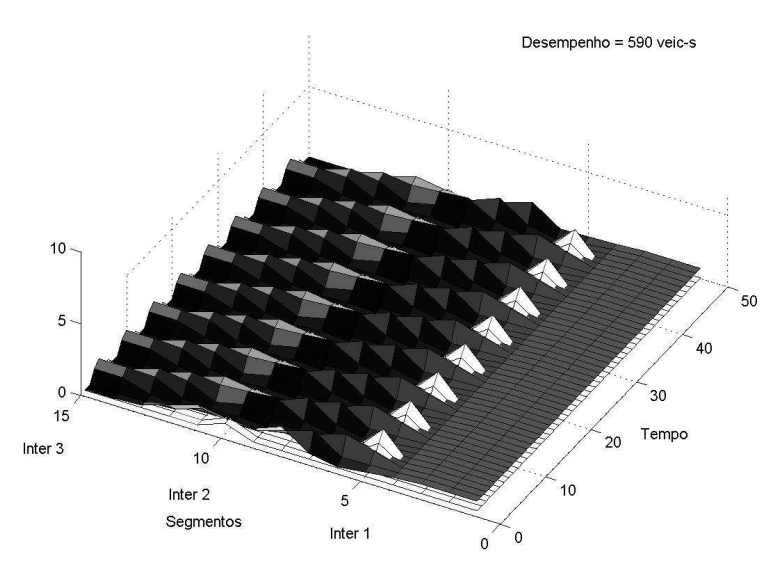

(a)

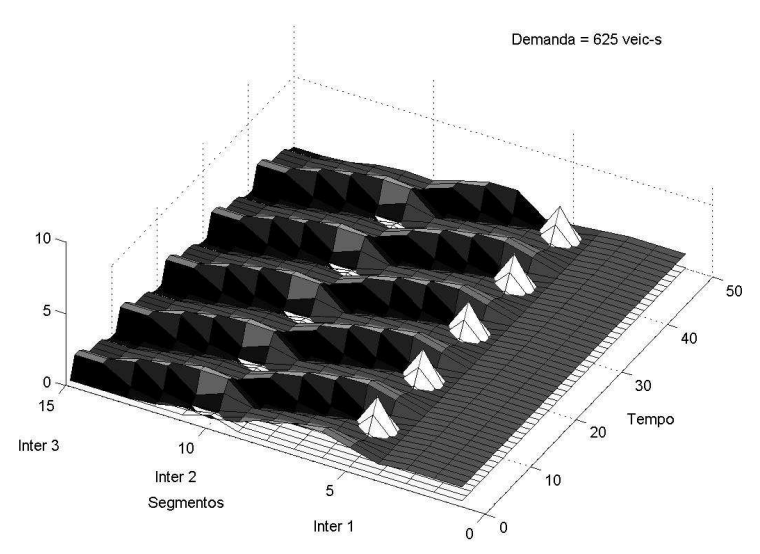

(c)

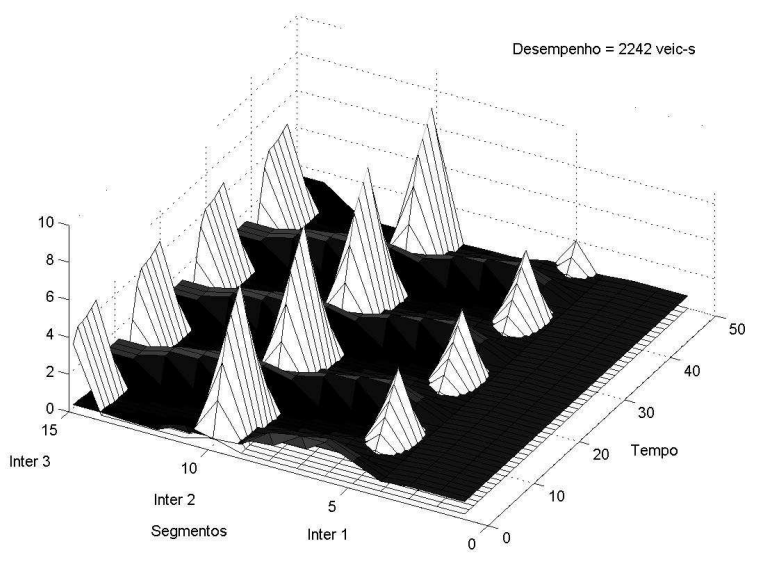

(b)

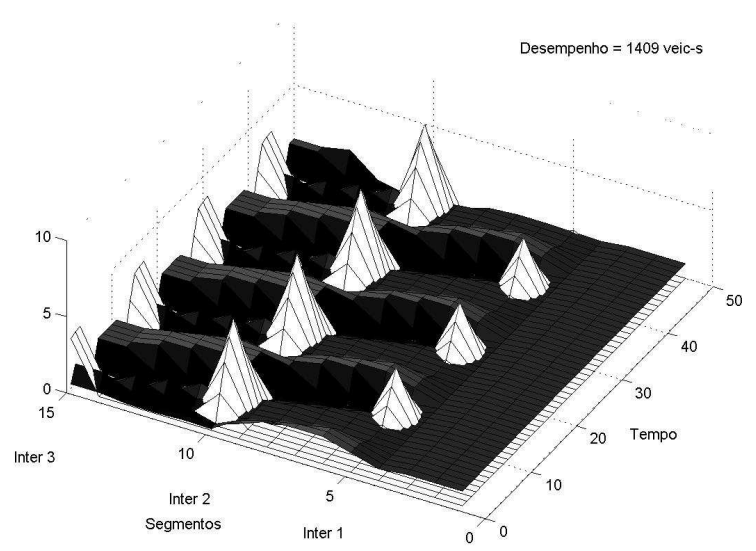

(d)

Figura 5: Comparação TRANSYT x algoritmo de descentralizado. 
Tabela 3: Valores de atraso obtidos com algoritmo descentralizado.

\begin{tabular}{lcccc}
\hline \hline Caso & Escada & Exp & Pulsada & Const \\
\hline BA & 12391 & 12002 & 8271 & 9426 \\
DA & 14352 & 13509 & 7812 & 11966 \\
BM & 5573 & 5498 & 3820 & 5193 \\
DM & 5050 & 5090 & 2026 & 5504 \\
\hline
\end{tabular}

e para as quatro situações de carregamento.

Os resultados apresentados na Tabela 3 mostram que, para um alto carregamento, o atraso aumenta em relação a um médio carregamento, para todas as distribuições de chegadas. Um resultado importante que pode ser verificado aparece para a distribuição pulsada onde ocorre uma melhoria significativa no desempenho do sistema. Esse é um resultado esperado, já que ocorrem períodos em que não chegam veículos e o algoritmo de controle adapta-se a esta situação. Isso sugere como perspectiva de estudos futuros um controle sobre os usuários que os mantivesse em pelotões.

São apresentados também resultados do algoritmo de controle em tempo real para a uma única distribuição de demanda a comparação é realizada entre as diferentes distribuições de chegadas. Essa avaliação tem como objetivo mostrar como o algoritmo reage às variações de demanda, mostrando assim sua robustez ao minimizar o atraso em diferentes situações de chegada.

A Figura 6 apresenta os resultados para diferentes distribuições de chegadas e um fluxo desbalanceado médio. Esta comparação proporciona a análise de como o algoritmo de controle reage às diferentes situações de tráfego. Também aqui ocorre a formação de vales caracterizando a descarga de pelotões nas interseções.
Pode ser verificado na Figura 6 que a maior formação de filas ocorre para as distribuições escada e exponencial (a) e (b), já que a predição do comportamento destas distribuições é dificultada pela natureza das chegadas. Já com chegadas constante e pulsada, o controle consegue rapidamente adequar-se e proporcionar um melhor desempenho.

\section{Discussões e Conclusões}

Foram apresentados os resultados de simulações com dois diferentes algoritmos de controle de tráfego. O resultado do algoritmo de tempo fixo foi apresentado para comparar o desempenho deste com o algoritmo desenvolvido neste trabalho. Foram analisados resultados referentes à progressão de pelotões nos links, e verificada uma boa coordenação, mesmo que implícita. Deve ser avaliada, em trabalhos futuros, a introdução de um critério de desempenho composto, que inclua o critério de número de paradas para verificar a influência na coordenação entre interseções.

Conclui-se que o algoritmo de controle desenvolvido neste trabalho apresentou melhores resultados do que o algoritmo ao qual foi comparado. No entanto, a questão da progressão de pelotões será mais explorada no futuro para obter-se melhor compreensão do comportamento do tráfego e do critério de otimização.

\section{Agradecimentos}

Os autores agradeçem a PROPPG-UEL e ao $\mathrm{CNPq}$ pelos financiamento das pesquisas.

\section{Referências}

CARLSON, R. C. Implementação de algoritmo para controle em tempo real de tráfego urbano. Projeto de Fim de Curso. Março 2004. 


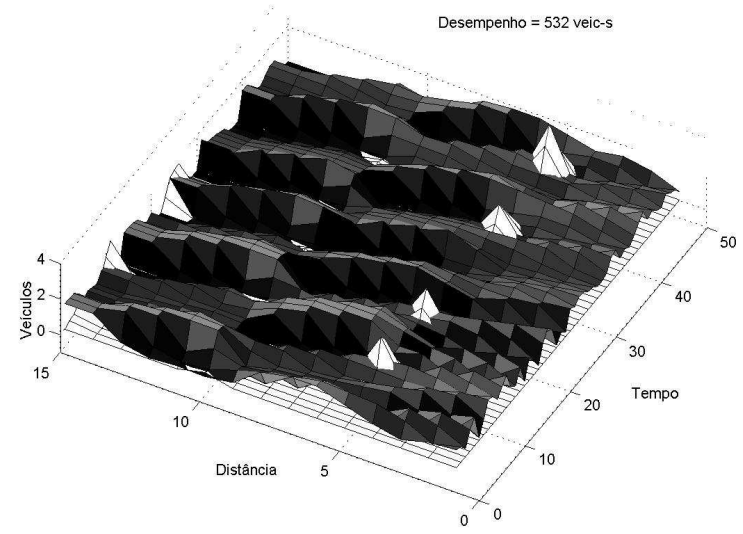

(a)- escada

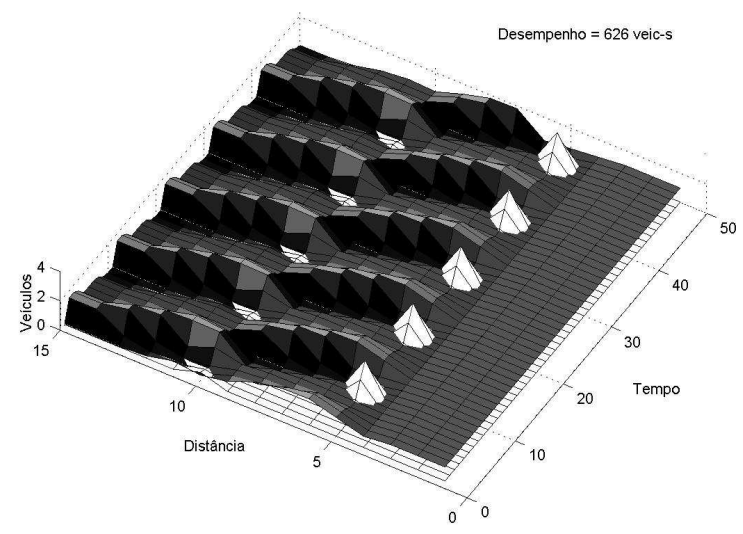

(c)- constante

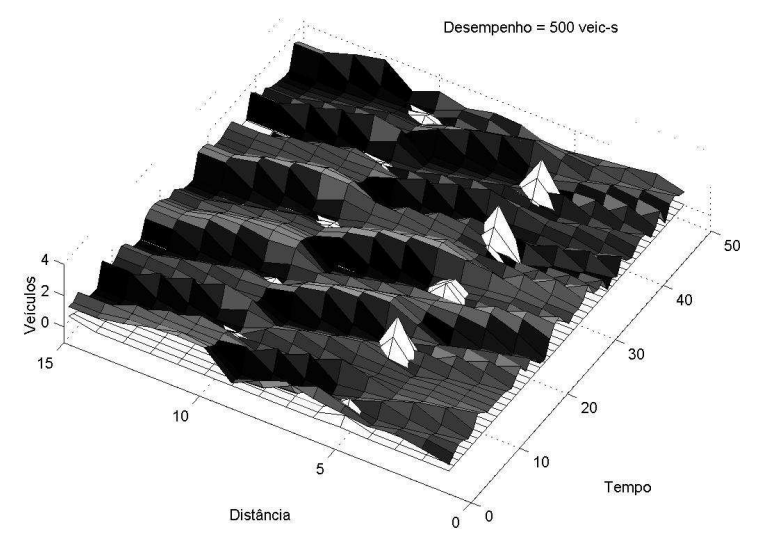

(b) - exponencial

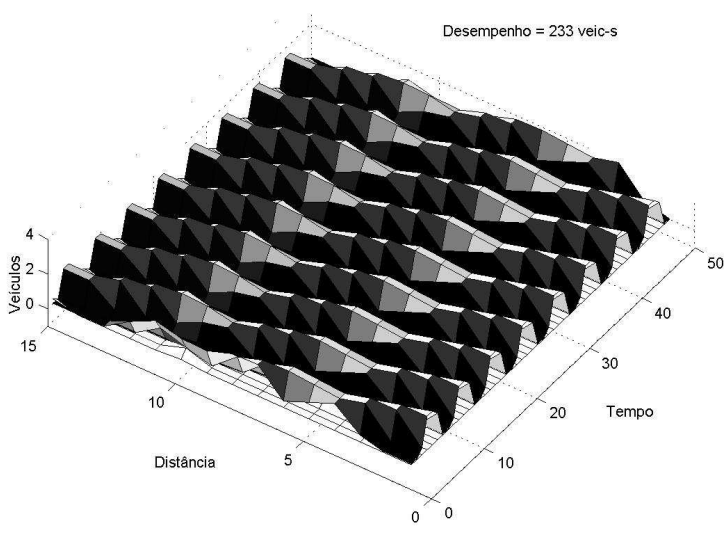

(d)- pulsada

Figura 6: Comparação entre diferentes distribuições de chegadas. 
CORMEN, T. H.; RIVEST, R. L.; STEIN, C.; LEISERSON, C. E. Algoritmos: Teoria e Prática. [S.l.]: Edição Americana, 2002.

FARGES, J. L.; KAMDEM, I.; LESORT, J. B. Realization and test of a prototype for real time urban traffic control. In: . [S.l.]: Drive Project V1022, 1991.

FARINES, J.; FRAGA, J. S.; OLIVEIRA, R. S. Sistemas de Tempo Real. São Paulo-SP: IME-USP, 2000.

GARTNER, N. H. Development and testing of a demand-responsive strategy for traffic signal control. In: AMERICAN CONTROL CONFERENCE. [S.I.], 1982. p. $578-583$.

GARTNER, N. H. OPAC: A demand responsive strategy for traffic signal control. In: TRANSPORTATION RESEARCH RECORD. [S.l.], 1983. v. 906, p. 75-81.

GERLOUGH, D. L.; HUBER, M. J. Traffic flow theory. Washington, D. C., n. Special Report, V. 165, 1975.

LOWRIE, P. SCATS: A traffic responsive method for controllig urban traffic. Sidney: NSW, 1990.

MIRCHANDANI, P.; HEAD, L. Rhodes: A realtime traffic signal control system: architecture, algorithms, and analysis. Transportation Research - Part $C$, New York, v. 8, p. 105-114, 2001.
NEWELL, G. F. The rolling horizon scheme of traffic signal control. Transportation Research. Part A, Elsevier Science Ltd., Great Britain, v. 32, p. 39-44, 1998.

PORCHE, I.; LAFORTUNE, S. Adaptive look-ahead optimization of traffic signals. 1997.

PORCHE, I.; SAMPATH, M.; SENGUPTA, R.; CHEN, Y. L.; LAFORTUNE, S. A decentralized scheme for real-time optimization of traffic signals. In: IEEE INTERNATIONAL CONFERENCE ON CONTROL APPLICATIONS. International Conference on Control Aplication, Dearbon, 1996. p. 582 589.

ROBERTSON, D. I. TRANSYT: A traffic network study tool. In: ROAD RESEARCH LABORATORY. Crowthorne, England, 1968.

ROBERTSON, D. I.; BRETHERTON, R. D. Optmization networks of traffic signals in real-time: the SCOOT method. IEEE Transactions on Vehicular Technology, v. 40, p. 11-15, 1991.

WILSHIRE, R.; BLACK, R.; GROCHOSKE, R.; HIGINBOTHAM, J. Traffic Control Systems Handbook. Special report - fhwa-ip-85-11. Washington D.C., 1985. 